\title{
Multimodality Medical Image Fusion Technique using Hybrid Method
}

\author{
Riddhi P. Shingadiya \\ Department of Computer Engineering \\ Parul Institute of Engineering and Technology, \\ India
}

\begin{abstract}
Multimodality medical image fusion is used to improve the imaging quality and reduce randomness and redundancy in order to increase the clinical applicability of medical images for diagnosis and assessment of medical problems. In this paper a hybrid method based on the non-subsampled contourlet transform (NSCT) and fractional fourier transform (FRFT) is proposed. CT \& MR images of different cases have been used to test the proposed method and results are compared with those of the other conventional image fusion methods by both visual analysis and quantitative analysis.
\end{abstract}

\section{Keywords}

Non-subsampled contourlet transform, fractional fourier transform, phase congruency and directive contrast.

\section{INTRODUCTION}

Medical image fusion is the process of combining multiple images from single or multiple imaging modalities to improve the imaging quality for clinical application. Multimodal medical image fusion algorithms have shown notable achievements in improving clinical accuracy. In this paper, two different imaging modalities CT and MRI are used for image fusion. Fusion of $\mathrm{CT}$ and MR images allows simultaneous visualization of details of bony anatomy provided by CT image and details of soft tissue anatomy provided by MR image. This helps the radiologist for the precise diagnosis of disease and for more effective interventional treatment procedures.

In this work, a hybrid medical image fusion method is presented by taking the complementary advantages of two powerful image representation theories: non-subsampled contourlet transform (NSCT) and fractional fourier transform (FRFT). The selection principles of the lowpass sub-band coefficients and the bandpass directional sub-band coefficients obtained after decomposition of images are discussed in detail, respectively. Objective evaluation index systems, such as mutual information, structural similarity based metric and edge based similarity measure are employed to judge quality of fused images, in addition to the visual analysis.

The rest of the paper is organized as follows. Section 2 reviews the theory of the FRFT \& NSCT in brief. Section 3 describes the proposed image fusion algorithm. Experimental results and discussion are given in Section 4. And the concluding remarks are presented in Section 5.

\author{
Rahul Joshi \\ Department of Information Technology \\ Parul Institute of Engineering and Technology, \\ India
}

\section{METHODS INVOLVED IN IMAGE FUSION}

\subsection{FRFT [12]}

Fourier transform is as transformations of a frequency domain signal into time domain signal. Apparently, fractional fourier transforms can transform a signal into the domain between time and frequency, it is a rotation in the time-frequency domain. FRFT is defined as:

$$
X p(u)=F p[X](u)=\int_{-\infty}^{+\infty} X(t) K p(t ; u) d t
$$

where $p$ is the order of FRFT, it can be any real number; $\alpha=\frac{p \Pi}{2}$ is the FRFT operator; $K p(t ; u)$ is the FRFT transform

kernel:

$K p(t, u)=\left\{\begin{array}{lc}\sqrt{(1-j \cot \alpha)} \exp j \prod & \left(t^{2} \cot \alpha\right. \\ \left.-2 u t \csc \alpha+u^{2} \cot \alpha\right), & \alpha \neq n \prod \\ \delta(t-u), & \alpha=2 n \prod \\ \delta(t+u), & \alpha=(2 n \pm 1) \Pi\end{array}\right.$

The inverse transform for FRFT:

$$
x(t) F_{-p}\left[x_{-p}\right](t)=\int_{-\infty}^{+\infty} X_{p}(u) K_{-p}(t-u) d u
$$

\subsection{NSCT}

NSCT computation framework proposed in [13], which has properties like multi-scale, multi-direction, localization and shift invariance. NSCT decomposition framework shown in fig.(1), which can be divided into two stages as described below:

2.2.1 Non-subsampled pyramid (NSP) structure: Three-stage non-subsampled pyramid decomposition is illustrated in fig.(2), which ensures the multi-scale property.

\subsubsection{Non-subsampled Directional Filterbank (NSDFB) structure:}

Four-channel non-subsampled directional filter bank constructed with two-channel fan filter banks structure is illustrated in fig.(3), which ensure the directionality. 


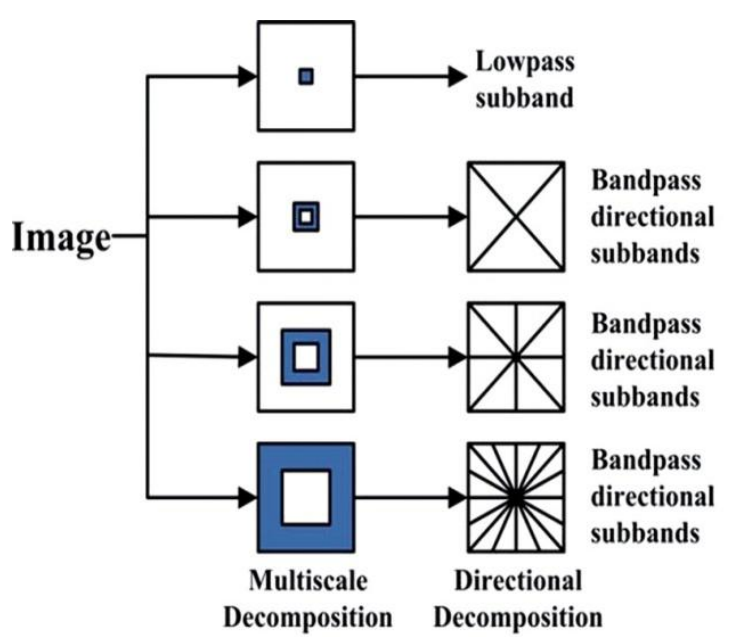

Fig 1: Decomposition framework of the NSCT [9]

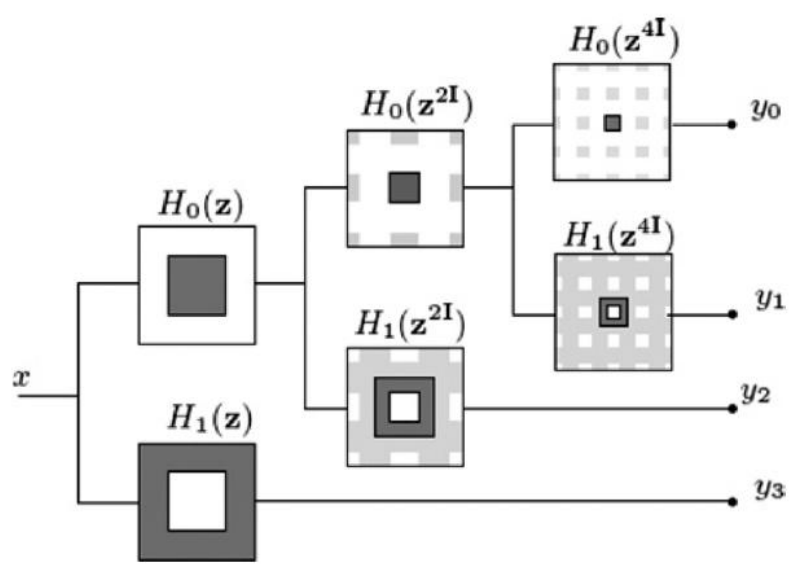

Fig 2: Three-stage non-subsampled pyramid decomposition [1]

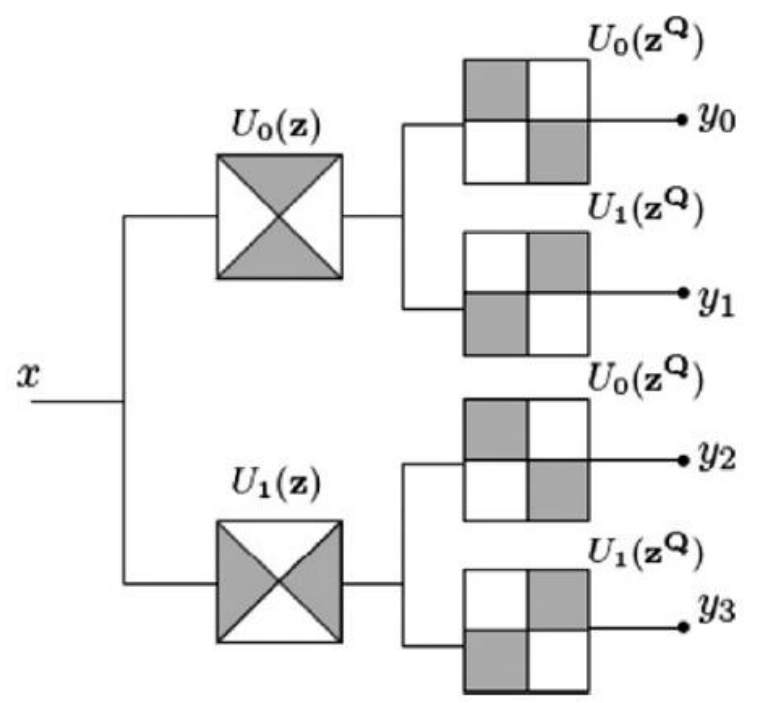

Fig 3: Four-channel non-subsampled directional filter bank [1]

\section{PROPOSED IMAGE FUSION METHOD}

The block diagram of proposed method is shown in fig.(4), in which FRFT is performed on source images. After that low frequency \& high frequency coefficient are obtained by performing NSCT decomposition. Low \& high coefficients are fused using different fusion rule. Finally, the fused image is constructed by the inverse NSCT \& inverse FRFT with all composite coefficients.

\subsection{Fusion rules}

Two different fusion rules based on phase congruency and directive contrast are used to fuse low- and high-frequency sub-bands.

\subsubsection{Phase Congruency:}

The phase congruency proposed in [1], is suggest that significant features can be found on points in the image, where the fourier components are maximally in phase. Phase congruency feature is invariant to illumination and contrast changes. Mathematically phase congruency is defined as :

$$
P_{x, y}^{o}=\frac{\sum_{n} W_{x, y}^{o}\left\lfloor A_{x, y}^{o, n}\left(\cos \left(\phi_{x, y}^{o, n}-\tilde{\phi}_{x, y}^{o}\right)-\mid \sin \left(\phi_{x, y}^{o, n}-\widetilde{\phi}_{x, y}^{o}\right)\right)-T\right\rfloor_{+}}{\sum_{n} A_{x, y}^{o, n}+\epsilon}
$$

Where, $W_{x, y}^{o}$ is the weight factor based on the frequency spread,

$A_{x, y}^{o, n} \& \phi_{x, y}^{o, n}$ are the amplitude and phase for the scale $\mathrm{n}$

$\tilde{\phi}_{x, y}^{o}$ is the weighted mean phase,

$\mathrm{T}$ is a noise threshold constant,

$\varepsilon$ is a small constant to avoid divisions by zero and the symbol []$+$ denotes that the enclosed quantity is equal to itself when the value is positive, and zero otherwise.

\subsubsection{Directive Contrast:}

The directive contrast proposed in [1], the sum-modifiedlaplacian is integrated with the directive contrast to produce accurate salient features. Mathematically directive contrast is defined as :

$$
D_{l, \theta}(i, j)=\left\{\begin{array}{lll}
\frac{S M L_{l, \theta}(i, j)}{I_{l}(i, j)}, & \text { if } & I_{l}(i, j) \neq 0 \\
S M L_{l, \theta}(i, j), & \text { if } & I_{l}(i, j)=0
\end{array}\right.
$$

Where $S M L_{l, \theta}$ is the sum-modified-laplacian of the NSCT frequency bands at scale $l$ and orientation $\theta$. On the other hand $\mathrm{I}_{1}(\mathrm{i}, \mathrm{j})$ is the low-frequency sub-band at the coarsest level (l).

\section{RESULT AND DISCUSSION}

Several experimental results are employed to demonstrate the great validity and feasibility of the proposed algorithm. Human visual perception can help judge the effects of fusion results. However, it is easily influenced by visual psychological factors. The effect of image fusion should be based on subjective vision and objective quantitative evaluation criteria. Some objective evaluation index systems [1], such as mutual information (MI), structural similarity based metric (Qs) and edge based similarity measure $(\mathrm{QAB} / \mathrm{F})$ are used. See [1] for the detailed implementation of the evaluation index systems. 


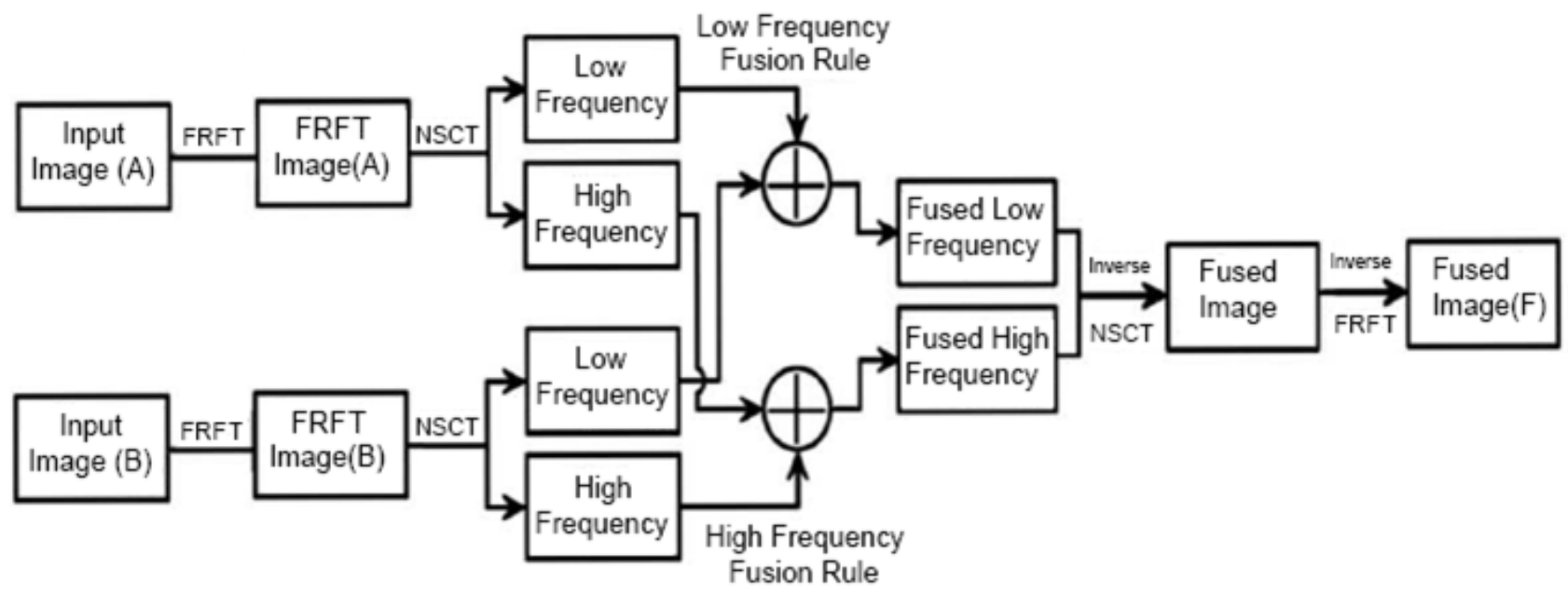

Fig 4: Block diagram of FRFT-NSCT

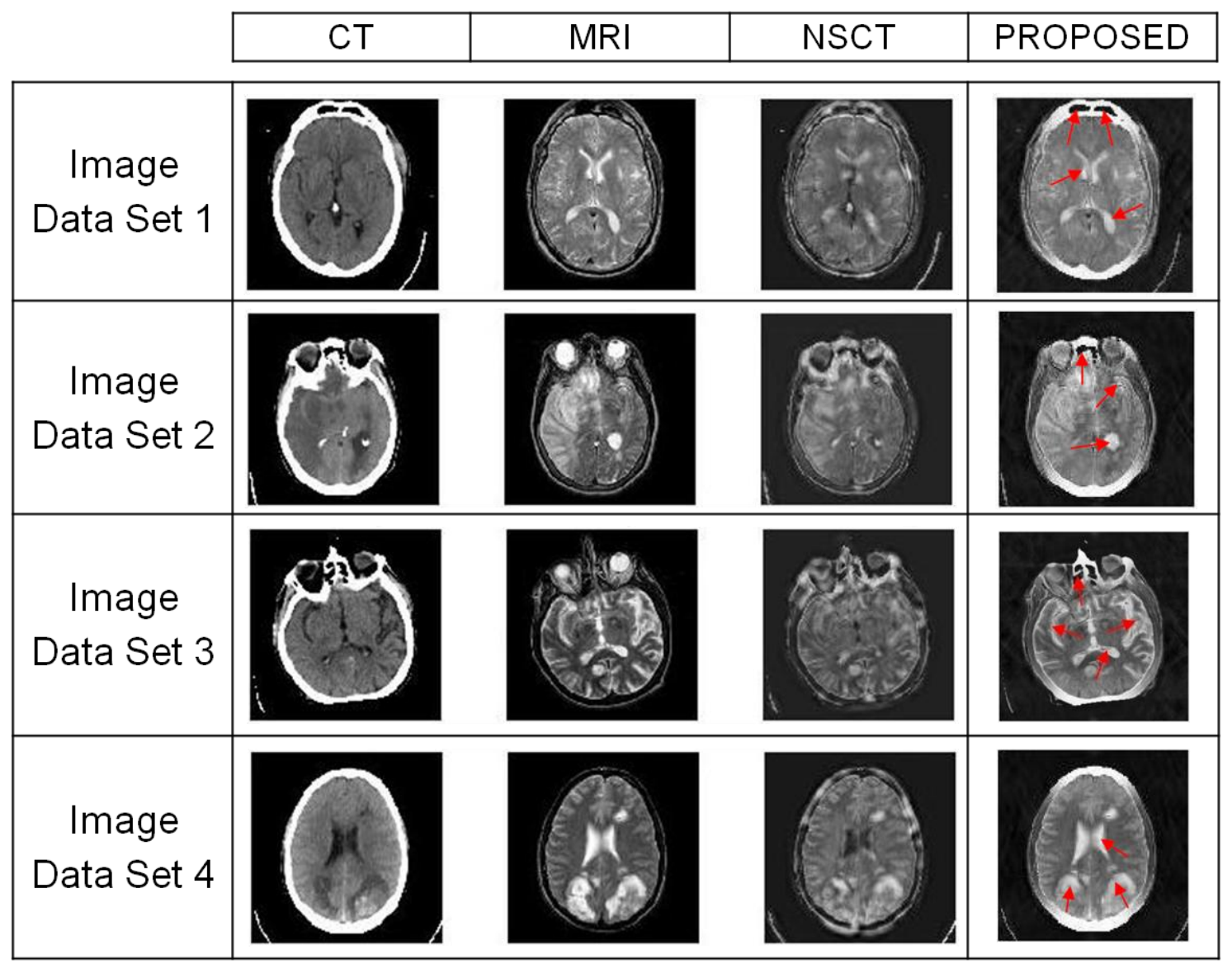

Fig 5: The multimodal medical image fusion results of NSCT[a] \& proposed method

CT \& MRI images of diseased person's brain with meningioma brain tumor, fatal stroke, multiple infarcts brain tumor and sarcoma brain tumor are considered to evaluate the performance of the proposed image fusion approach.

Data sets given in fig.(5) are brain images of

1) Meningioma brain tumor,

2) Fatal stroke,

3) Multiple infarcts brain tumor and
4) Sarcoma brain tumor.

For all these images, result of proposed framework are compared with the non-subsample contourlet transform method of [1]. 
Table 1. Statistical result of fused images

\begin{tabular}{|c|c|c|c|}
\hline Images Modalities & Indices & NSCT & Proposed \\
\hline Image Dataset 1 & MI & 1.0850 & 1.5949 \\
(MRI and CT) & $\mathbf{Q s}$ & 0.7970 & 0.7967 \\
& $\mathbf{Q B} / \mathbf{F}$ & 0.6952 & 0.7768 \\
\hline \multirow{2}{*}{ Image Dataset 2 } & MI & 1.1759 & 1.4923 \\
(MRI and CT) & $\mathbf{Q s}$ & 0.7965 & 0.8162 \\
& $\mathbf{Q A B} / \mathbf{F}$ & 0.6938 & 0.7758 \\
\hline \multirow{2}{*}{ Image Dataset 3 } & $\mathbf{M I}$ & 1.1242 & 1.5331 \\
(MRI and CT) & $\mathbf{Q s}$ & 0.7551 & 0.7792 \\
& $\mathbf{Q A B / F}$ & 0.6425 & 0.7799 \\
\hline \multirow{2}{*}{ Image Dataset 4 } & $\mathbf{M I}$ & 1.2255 & 1.4797 \\
(MRI and CT) & $\mathbf{Q s}$ & 0.8243 & 0.8181 \\
& $\mathbf{Q A B / F}$ & 0.6734 & 0.7953 \\
\hline
\end{tabular}

\section{CONCLUSION}

In this paper, a hybrid medical image fusion method is presented by taking the complementary advantages of two powerful image representation theories: non-subsampled contourlet transform and fractional fourier transform. NSCT limits the signal analysis to frequency domain, which is over come in enhancement work with the help of fractional fourier transform. Fractional fourier transform develops the signal analysis to fractional domain. So that hybrid image fusion algorithm has merits of both NSCT \& fractional fourier transform. Other important part is fusion rules which are adopted as below. The two different fusion rules based on phase congruency and directive contrast are used to fuse lowand high-frequency sub-bands.

In order to show the practical applicability of the proposed method, CT \& MRI images of diseased person's brain with meningioma brain tumor, fatal stroke, multiple infarcts brain tumor and sarcoma brain tumor are considered to evaluate the performance of the proposed image fusion approach. A statistical \& visual results of experiment shows the superiority of the proposed methods over NSCT [1].

\section{REFERENCES}

[1] Bhatnagar, Gaurav, Q. M. Wu, and Zheng Liu. "Directive contrast based multimodal medical image fusion in NSCT domain." Multimedia, IEEE Transactions on 15.5 (2013): 1014-1024 [2] K. Song and J. W. Ji, "An image fusion algorithm
based on the wavelet transform," Journal of Shen Yang Agricultural University,vol. 38, pp. 845-848, 2007
[3] C. Gargour, M. Gabrea, V. Ramachandran, and J. M Lina, "A Short introduction to wavelets and their applications," IEEE Circuits and Systems Magazine, vol. 9, no. 2, pp. 57-68, 2009

[4] G. Pajares and J. M. de la Cruz, "A wavelet based image fusion tutorial," Pattern Recognition, vol. 37, no. 9, pp. 1855-1872, 2004

[5] D. Y. Qin, J. D. Wang, and P. Li, "Wavelet base selection and evaluation for image fusion," optoelectronic Technology, vol. 26, pp. 203-207, 2006.

[6] F. Nencini, A. Garzelli, S. Baronti, and L. Alparone, "Remot sensing image fusion using the curvelet transform," Information Fusion, vol. 8, no. 2, pp. 143156, 2007.

[7] X. Qu, J. Yan, H. Xiao, and Z. Zhu, "Image fusion algorithm based on spatial frequency-motivated pulse coupled neural networks in nonsubsampled contourlet transform domain," Acta Automatica Sinica, vol.34, no. 12, pp. 1508-1514, 2008.

[8] Xing Xiaoxue,Lei Yanmin, "Medical Image Fusion in Compressed Sensing Based on Non-subsampled Contourlet Transform"2013 IEEE.

[9] Y. Chai, H. Li, and X. Zhang, "Multifocus image fusion based on features contrast of multiscale products in nonsubsampled contourlet transform domain," Optik, vol. 123, pp. 569-581, 2012.

[10] C. Yang, J. Zhang, X. Wang, and X. Liu, "A novel similarity based quality metric for image fusion," Inf. Fusion, vol. 9, pp. 156-160, 2008.

[11] Peiguang Wang, Hua Tian, and Wei Zheng," A Novel Image Fusion Method Based on FRFT- NSCT" Hindawi Publishing Corporation, Volume 2013.

[12] Sang, Gaoli, et al. "A fractional Fourier transform based method of image fusion." Image and Signal Processing (CISP), 2013 6th International Congress on. Vol. 2. IEEE, 2013.

[13] Da Cunha, Arthur L., Jianping Zhou, and Minh N. Do. "The nonsubsampled contourlet transform: theory, design, and applications." Image Processing, IEEE Transactions on 15.10 (2006): 3089-3101. 